\title{
Digitale Kommunikation im Gesundheitswesen
}

\section{Autor: Hans-Hermann Dirksen}

\section{ZUSAMMENFASSUNG}

Ein Artikel über rechtliche Fragen der digitalen Kommunikation in einer Zeitschrift der Erfahrungsheilkunde mag zunächst vielleicht überraschen. Aber auch eine Heilkunde, die sich der Erfahrung sowie der Tradition verpflichtet fühlt, beruht auf einem grundsätzlich zeitlosen Aspekt, nämlich der guten Kommunikation zwischen Behandler und Patienten. Der Artikel möchte daher aufzeigen, welche Aspekte zu berücksichtigen sind, wenn sich das Kommunikationsverhalten einer neuen digitalen Zeit zuwendet und damit Patienten und Behandler vor besondere Herausforderungen stellt.

\section{Schlüsselwörter}

Digitale Kommunikation, Patientenaufklärung, Datenschutz

\section{ABSTRACT}

At first, an article on legal issues of digital communication in a journal of Erfahrungsheilkunde may be a surprise. But even medicine which is committed to experience and tradition is based on a fundamentally timeless aspect, namely the good communication between practitioner and patient. Therefore, the article wants to point out which aspects have to be taken into consideration when the communication behavior turns towards a new digital age and thus poses special challenges for patients and practitioners.

Keywords

Digital communication, patient education, data protection

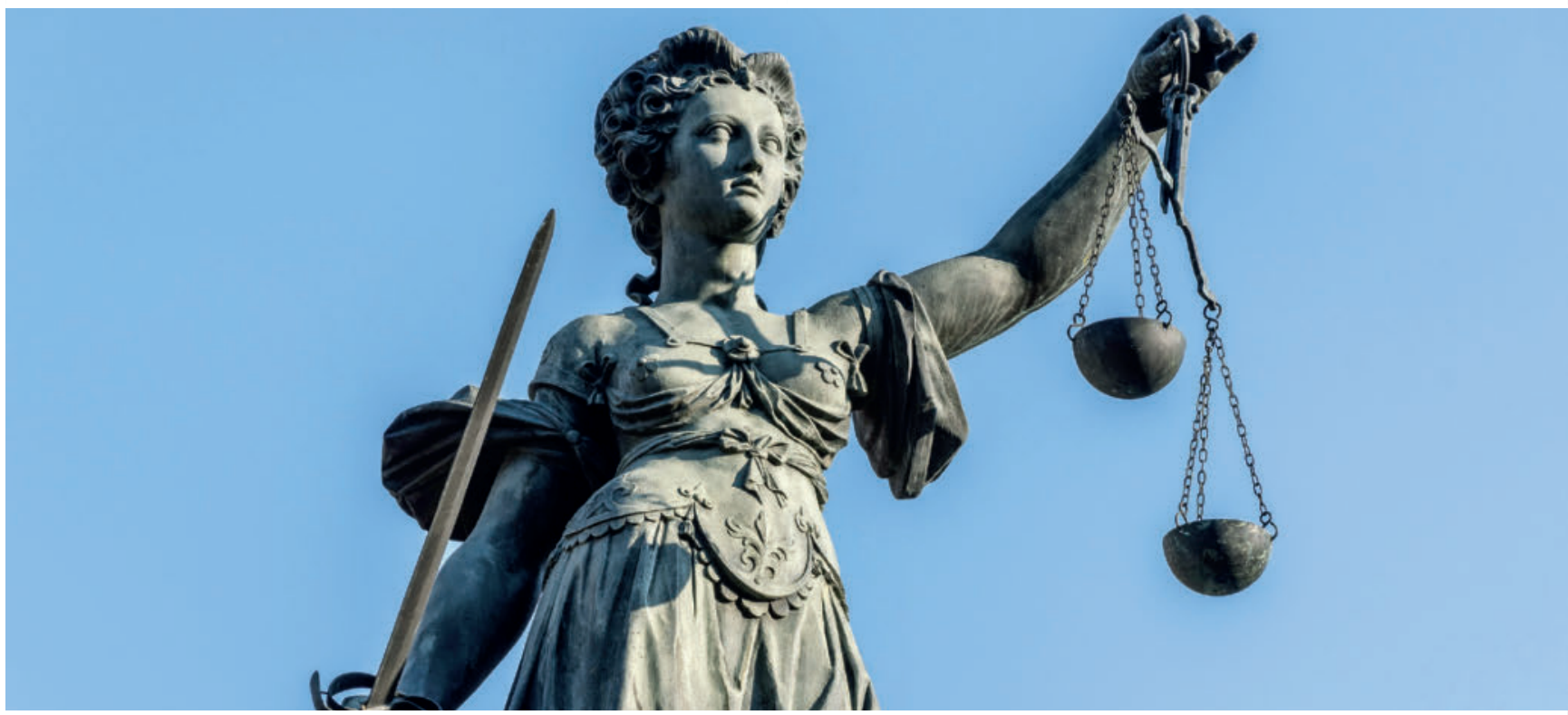

Foto: helmutvogler/Adobe Stock

\section{Gegenstand der digitalen \\ Kommunikation im Gesundheitswesen}

Viele Behandler, Praxen und Kliniken nutzen heute bereits ganz selbstverständlich digitale Anwendungen in der täglichen Routine. Sie haben ihre Patientendokumentation digitalisiert, und auch Terminplanung und Wartezimmermanagement sind bei vielen Praxen weitgehend digitalisiert. Viele Hausärzte nutzen digitale Anwendungen, um Arzneimittelwechselwirkungen zu erkennen. Praxen und Kliniken digitalisieren ihre analogen Daten nachträglich zu Aufbewahrungszwecken. Aufklärungsgespräche werden mit Unterstützung von digitalen Informationsbögen geführt und Einwilligungserklärungen auf dem Tablet unterschrieben [6].

Die Möglichkeiten digitaler Kommunikation sind folglich umfangreich und wenn gut umgesetzt patientenzentriert. Patienten können besser informiert und unterstützt werden, chronische Krankheitsverläufe besser begleitet und der Patient kann mehr ins Zentrum der Behandlung gerückt werden. Aber um das alles umzusetzen, muss die Kommunikation als solche funktionieren.

Infolge der Digitalisierung kommt es schon seit Längerem zu einer starken Zunahme digital ausgetauschter Kommunikation mittels E-Mails, neuerdings auch durch Instant 
Messaging wie Telegram, Threema oder WhatsApp oder auch durch Bildübertragungsdienste wie Skype.

Praxen kommunizieren mit Patienten mindestens zur Hälfte auf digitalem Wege, wobei sich ein altersabhängiges Gefälle zeigt, wonach erwartungsgemäß jüngere Ärzte/Psychotherapeuten öfter digital kommunizieren [6]. Unter den möglichen Formen der digitalen Kommunikation dominiert dabei die E-Mail, die von über der Hälfte der Praxen hierfür genutzt wird. Messenger-Dienste bzw. SMS folgen in der Nutzungshäufigkeit dagegen erst mit deutlichem Abstand [6].

Untersuchungen zeigen zudem, dass sich immer mehr Patienten über Symptome, Diagnose und Behandlungsmöglichkeiten im Internet informieren [10]. Es dürfte bald allgemeiner Standard sein, dass sich Patienten vor oder nach dem Arztbesuch über die Erkrankung und Therapiemaßnahmen selbst informieren und gezielte Fragen an ihren Arzt stellen.

Natürlich wird die Asymmetrie im Fachwissen zwischen Arzt und Patienten dadurch nie völlig aufgelöst werden, und das ist auch in Ordnung. Patienten sind jedoch immer mehr in der Lage, die Ausführungen ihres Arztes zu verstehen und als Konsequenz ihre eigene Therapie besser zu unterstützen. Dadurch entsteht in den letzten Jahren mehr und mehr der informierte Patient, der demgemäß tatsächlich auf so etwas wie Augenhöhe mit seinem Behandler kommen könnte. Dies verändert die Gesprächsführungen und die Anforderungen an die Aufklärung enorm.

\section{Digitale Kommunikation und Patientenaufklärung}

Die konkrete Patienteninformation durch den Arzt spielt auch vor dem Hintergrund des Arzthaftungsrechts eine entscheidende Rolle im Arzt-Patienten-Verhältnis. Der Arzt ist gehalten, den Patienten auch bzw. gerade in Zeiten der Digitalisierung bei der Ausübung seines Selbstbestimmungsrechts zu unterstützen und ihm zu helfen, die medizinischen Informationen zu verarbeiten und zu bewerten. Nur bei entsprechender Patienten-Compliance können beide von den neuen technischen Entwicklungen profitieren.

Nach wie vor muss gem. §630e Abs. 2 Nr. 1 BGB die Aufklärung eines Patienten in Deutschland mündlich erfolgen. Eine Aufklärung nur per E-Mail ist nicht möglich. Schriftliche Aufklärungsbögen dürfen zusätzlich ausgeteilt werden und können die mündliche Aufklärung unterstützen. Der Arzt hat sich aber dennoch in einem persönlichen Gespräch stets davon zu überzeugen, ob der Patient die Informationen verstanden hat und ob er noch weitere Informationen wünscht [11].
Die Aufklärung eines Patienten

muss in Deutschland nach wie vor

in einem persönlichen Gespräch

erfolgen.

Gemäß §630e Abs. 2 Satz 2 BGB sind dem Patienten Abschriften von Unterlagen, die er im Zusammenhang mit der Aufklärung oder Einwilligung unterzeichnet hat, auszuhändigen. Dies wird in der Regel immer noch durch Kopien vorgenommen werden müssen.

In $\S 630$ c Abs. 2 Satz 1 BGB und $\S 630$ e Abs. 1 und Abs. 2 Satz 1 Nr. 1 BGB ist des Weiteren festgelegt, dass die Aufklärung durch den Behandler zu erfolgen hat. Hieraus ergibt sich, dass die Aufklärung über medizinische Eingriffe nicht an nichtärztliches Personal delegiert bzw. von außenstehenden Dienstleistern durchgeführt werden darf. Auch ist es nicht ausreichend, wenn die Aufklärung durch nichtärztliches Personal im Beisein eines Arztes erfolgt [12]. Die Aufklärung muss in jedem Fall durch einen entsprechend ausgebildeten Arzt vorgenommen werden.

In einfach gelagerten Fällen kann eine Aufklärung auch telefonisch erfolgen. Dies jedoch nur, wenn dem Behandler der Patient bereits persönlich bekannt ist [13]. Ebenso ist es auch möglich, die Aufklärung via Videochat vorzunehmen. Dies soll jedoch die Ausnahme bleiben und ist nur in besonders gelagerten Fällen zulässig. Grundsätzlich ist stets ein persönliches Gespräch zwischen Behandler und Patient erforderlich [14].

Allerdings ist es nicht erforderlich, dass stets derjenige Arzt die Aufklärung vornimmt, der später auch die Behandlung durchführt. Insoweit ist es grundsätzlich möglich, die Aufklärung an einen Kollegen zu delegieren. Notwendige Voraussetzung ist jedoch in jedem Fall, dass dieser Kollege über die zur Durchführung der konkreten Behandlung notwendige Ausbildung verfügt.

\section{Digitale Unterstützungsmöglichkeiten}

Die Patienten möchten die persönliche Wartezeit verkürzen und deshalb auf elektronischem Wege einen Termin vereinbaren oder am besten sofort mit dem Arzt ihres Vertrauens sprechen. Tatsächlich fordern daher immer mehr Patienten, dass Behandler auch diese neuen Möglichkeiten der digitalen Technik nutzen. Daher ist es erforderlich, dass sich Behandler über Möglichkeiten der neuen digitalen Kommunikation informieren und sich damit auseinandersetzen.

Das Angebot von digitalen bzw. Online-Services der Praxen hält sich gegenwärtig allerdings noch in Grenzen. Nur wenige Praxen bieten die Möglichkeit für eine Online-Terminvereinbarung oder die Bereitstellung eines Medikationsplans für den Patienten, und ebenfalls bieten nur wenige 
der Praxen an, Unterlagen aus der Patientendokumentation digital zur Verfügung zu stellen oder online ein Wiederholungsrezept zu bestellen. Dagegen findet man bei deutlich mehr Praxen keine solchen digitalen Service-Angebote für Patienten [6].

Die sich in diesem Feld der digitalen Kommunikation ergebenden Chancen für ein gutes Praxismarketing insbesondere auch durch die Nutzung der sozialen Medien wie Facebook, Instagram oder Twitter sind enorm, die Risiken nicht zu übersehen, aber lösbar. Rechtsverstöße, Abmahnungen sowie gerichtliche Auseinandersetzungen sind kosten- und zeitraubende Angelegenheiten, die in den meisten Fällen mit fachkundiger Hilfestellung vermieden werden könnten. Daher ist es grundsätzlich empfehlenswert, die Geschäfts- und Nutzungsbedingungen einzelner Webangebote aufmerksam zu lesen, um zu verhindern, dass man unter Umständen unwissentlich dem Verzicht auf bestimmte Verwertungs- und Schutzrechte zustimmt.

Es ist Behandlern daher sehr zu empfehlen, informierte Rücksprache z. B. mit einem Rechtsanwalt zu nehmen und die konkrete Gestaltung eines Social-Media-Engagements mit seinen Optionen abzuklären.

\section{Einsatz von Gesundheits-Apps}

Digitalisierung ermöglicht ebenfalls eine sehr engmaschige und individualisierte Unterstützung und Betreuung. Fitnesstracker können sportliche Aktivitäten unterstützen und in Präventionsprogramme eingebaut werden, telemedizinische Dienste können Therapien unterstützen, auch wenn Patienten physisch nicht in der Praxis oder im Krankenhaus sind, Teleangebote für Rehamaßnahmen können zur Wiederherstellung körperlicher und kognitiver Beeinträchtigungen genutzt werden. Das Anwendungsspektrum digitaler Technologien ist insofern sehr groß und umfasst alle gesundheitlichen Bereiche: Prävention, Diagnose, Therapie und Rehabilitation. Dabei können die Angebote auf verschiedene Zielgruppen hin ausgerichtet werden und damit eine Diversifizierung in der gesundheitlichen Versorgung unterstützen.

Dass Patienten ihre Ärzte/Psychotherapeuten mit selbst erhobenen digitalen Daten über ihre Gesundheit (z. B. Pulswerte aus Apps, Daten aus Fitnesstrackern) konfrontieren, kommt im Praxisalltag allerdings bislang selten vor. Nur wenige Praxen berichten, dass zumindest ein geringer Patientenanteil solche Daten im Gespräch mit dem Behandler zeigt. Am häufigsten geschieht dies noch in den Hausarztpraxen, von denen es rund ein Viertel mit einem mindestens geringen, teilweise auch größeren Patientenanteil zu tun hat, der selbst erhobene Daten bei der Behandlung vorzeigt [6].

Die Praxen bewerten Informationen aus digitalen Gesundheitsdaten, die Patienten selbst erhoben haben, teilwei- se durchaus als hilfreich. Dieser Anteil variiert aber deutlich zwischen den Fachgruppen. Am offensten gegenüber selbst generierten digitalen Daten der Patienten sind die Hausärzte, von denen rund zwei Drittel solche als sehr, eher oder teilweise hilfreich ansehen, gefolgt von den interdisziplinär besetzten Praxen [6].

\section{Patientendatenschutz bei der digitalen Kommunikation}

Im Zuge aktueller Digitalisierungsprozesse des Gesundheitswesens erweitern sich die Möglichkeiten der Datenerhebung und -auswertung enorm. In einer individualisierten Gesellschaft entstehen so neue Potenziale, um den pluralisierten gesundheitlichen Bedarfen von Individuen und Organisationen nachzukommen. Angesichts des Umstandes, dass es sich bei der digitalen Kommunikation im Gesundheitswesen um besonders sensible Daten, die zudem der ärztlichen Schweigepflicht unterliegen, handelt, müssen hier in besonderem Maß die Patientenrechte und der Datenschutz beachtet werden.

\section{Bei der digitalen Kommunikation im Gesundheitswesen müssen in besonderem Maße Patientenrechte und Datenschutz beachtet werden.}

Der Gesetzgeber spricht dem Patienten stets die vollständige Verfügungsgewalt über dessen personenbezogene (Gesundheits-)Daten zu. Das Selbstbestimmungsrecht der Patienten rückt damit auch im medizinischen Umfeld mehr und mehr in den Mittelpunkt.

Ist beispielsweise die Verwendung der personenbezogenen Daten eines Patienten zwingend zur Erfüllung des Behandlungsvertrages erforderlich, dann darf der Behandler die Daten zu dem entsprechenden Zweck gem. Art. 6 Abs. 1 b DSGVO verwenden, so beispielsweise die Adressdaten zur Übersendung von Briefen oder die Telefonnummer, um den Behandlungstermin zu vereinbaren. Sofern die Datennutzung über diesen Vertragszweck hinausgeht, dürfte der Behandler die Daten nur verwenden, wenn der Patient dazu eingewilligt hätte, wie etwa zur Veröffentlichung einer Bildergalerie der Teilnehmer eines Sommerfestes der Praxis. Die gleiche Frage stellt sich bei der Weitergabe von Daten. Ist eine Weitergabe zur Erfüllung des Vertragszweckes erforderlich, darf dieses geschehen, ansonsten nur mit konkreter Einwilligung.

Daher erlangt die Digitalisierung auch im Arzthaftungsrecht große Bedeutung. Ärzte müssen bei dem Umgang mit digitalen Anwendungen immer berücksichtigen, welche Haftungsrisiken aus ihnen erwachsen. Die einzelne Arztpraxis wird die internen Abläufe entsprechend anpassen müssen. Über die elektronische Kommunikation eingehende Arztbriefe und Informationen müssen von dem Arzt 
auch tatsächlich (zeitnah) zur Kenntnis genommen werden. Medikationspläne sind stets zu aktualisieren (§31 a Abs. 3 SGB V), ebenso wie die Notfalldaten [9].

Dabei ist es nach dem Willen des Gesetzgebers letztlich immer der Patient, der die Hoheit über die Daten ausübt und entscheidet, ob und welche Daten in der Akte gespeichert oder auch gelöscht werden. Die bekannte und von $\S 630$ f BGB gesetzlich vorgeschriebene Behandlungsdokumentation wird hierdurch nicht ersetzt.

\section{Neue Kommunikationswege im Gesundheitswesen}

Digitalisierung und Vernetzung machen es technisch ebenfalls möglich, den klassischen Arzt-Patienten-Kontakt durch den Einsatz von Fernkommunikationsmitteln wie Smartphone und Internet zu ergänzen oder gar zu ersetzen. Die damit verbundenen arzthaftungsrechtlichen Risiken sind nicht zu unterschätzen.

Telemedizinische Lösungen erlauben die Auflösung von Zeit und Raum, d. h., eine Diagnose oder Beurteilung einer Therapie kann erfolgen, ohne dass Behandler und Patienten zum gleichen Zeitpunkt im gleichen Raum sein müssen. Zwar ist der direkte menschliche Patientenkontakt in der Betreuung unbestritten besonders wichtig, eine begleitende telemedizinische Versorgung kann aber Behandlungen ergänzen und Patienten von möglicherweise langen Wegen oder Wartezeiten entlasten. Aus medizinisch-pflegerischer Sicht können telemedizinische Behandlungsformen die Qualität der Versorgung steigern, da eine engmaschigere Betreuung (z. B. durch eine Überwachung von Vitalparametern) aufgebaut werden kann als bei einer auf rein persönlichem Kontakt beruhenden Behandlung.

Ärzten war es lange berufsrechtlich verboten, Patienten ausschließlich über Kommunikationsmedien zu behandeln. Mindestens ein initialer persönlicher Kontakt analog und offline zwischen Arzt und Patient war unabdingbar. Im Mai vergangenen Jahres hat der 121. Deutsche Ärztetag in Erfurt jedoch eine Neufassung des hierfür maßgeblichen $§ 7$ Absatz 4 der Musterberufsordnung der Ärztinnen und Ärzte beschlossen, sodass nunmehr (nach Umsetzung durch die Landesärztekammern) eine ärztliche Behandlung unter bestimmten Voraussetzungen auch ausschließlich über Kommunikationsmedien erlaubt ist [7].

Diese für die Fernbehandlung ausschlaggebende Formulierung lautet nunmehr wie folgt: „Eine ausschließliche Beratung oder Behandlung über Kommunikationsmedien ist im Einzelfall erlaubt, wenn dies ärztlich vertretbar ist und die erforderliche ärztliche Sorgfalt insbesondere durch die Art und Weise der Befunderhebung, Beratung, Behandlung sowie Dokumentation gewahrt wird und die Patientin oder der Patient auch über die Besonderheiten der ausschließlichen Beratung und Behandlung über Kommunikations- medien aufgeklärt wird. “ Ob diese sehr begrüßenswerte Formulierung der Telemedizin zum allgemeinen Durchbruch verhilft, wird allerdings erst die Zukunft zeigen.

Bei allen Erwartungen, die in diese neuen Möglichkeiten des Arzt-Patienten-Kontakts mittels Kommunikationsmittel gesetzt werden, darf nicht vergessen werden, dass jede ärztliche Behandlung „nach den zum Zeitpunkt der Behandlung bestehenden, allgemein anerkannten fachlichen Standards zu erfolgen“ hat (§ 630a Absatz 2 BGB), und zwar in jeder Behandlungsphase. Auf den formalen Facharztstatus des behandelnden Arztes kommt es dabei zwar nicht an. Jedoch muss der Behandelnde über die tatsächliche fachliche Befähigung auf der Basis theoretischer Kenntnisse und praktischer Erfahrungen verfügen. Ein dieses Niveau nicht erreichender Arzt muss von einem erfahrenen Kollegen überwacht werden. Bleibt die Behandlung hinter dem Facharztstandard zurück, liegt ein Behandlungsfehler vor und es drohen sowohl zivilrechtliche als auch strafrechtliche Konsequenzen.

Der Facharztstandard gilt natürlich auch bei telemedizinischer Behandlung. Die gesamte Behandlung muss dem Facharztstandard genügen. Dass etwa in einem telemedizinischen Call-Center oder in einer an ein Telemedizinnetzwerk angeschlossenen Arztpraxis ein Facharzt sitzt, genügt dem nicht ohne Weiteres. Denn dieser kann nur auf Grundlage der ihm übermittelten Informationen tätig werden. Sind aber schon diese nicht ordnungsgemäß, d. h. dem Facharztstandard entsprechend erhoben, übermittelt und dargestellt, genügt die Behandlung insgesamt nicht mehr dem Facharztstandard. Haftungsrisiken können sich hier schon ganz am Anfang, bei der Befunderhebung ergeben.

Telemedizinische Angebote bringen zwangsläufig den Einsatz von Technik mit sich. Anders als bei klassischer Medizintechnik, die hohen Anforderungen und strengen Kontrollen bei Herstellung und Vertrieb unterliegt, kommt dabei auch und insbesondere „Consumer“-Hardware zum Einsatz, namentlich das Smartphone. Und auch das Internet als regelmäßiges Übertragungsmedium ist nicht auf hochverfügbare, zeitkritische und manipulationsgeschützte Kommunikation ausgelegt.

Was gilt, wenn die Technik versagt? Wenn etwa auf dem Übertragungswege wichtige Informationen verloren gehen oder gar vorsätzlich manipuliert werden? Sofern der Arzt auf unplausible Daten stößt, muss er dem nachgehen. Nötigenfalls muss er die telemedizinische Behandlung abbrechen und den Patienten nachdrücklich zur persönlichen Vorstellung in einer Praxis raten. Dasselbe gilt, wenn der Arzt während der Behandlung feststellt bzw. bei pflichtgemäßer Sorgfalt hätte feststellen müssen, dass die technischen Voraussetzungen für eine dem Facharztstandard genügende Behandlung nicht mehr bestehen. Noch ungeklärt ist dagegen, wie mit nicht erkennbaren Anwendungs-, Erhebungs- oder Übermittlungsfehlern umzugehen ist. 
Grundsätzlich ist kein Arzt verpflichtet, eine Behandlung zu übernehmen. Sobald die Übernahme jedoch vereinbart wurde, schuldet er folgerichtig eine facharztstandardgemäße Behandlung. In Anlehnung daran bedeutet das Bereithalten von einem telemedizinischen Angebot, dass das Risiko des Übernahmeverschuldens bestehen bleibt.

\section{Zusammenfassung}

Die digitale Kommunikation im Gesundheitswesen bedeutet eine Veränderung der regelmäßigen Abläufe und wiederkehrenden Prozesse durch die aktuelle technische Entwicklung. Aspekte der Datensicherheit und das Selbstbestimmungsrecht der Patienten stehen dabei im Mittelpunkt. Der Gesetzgeber selbst treibt die Entwicklung der Digitalisierung voran, neben den bestehenden Regelungen werden in der laufenden Legislaturperiode weitere Neuerungen folgen. Die Digitalisierung wird die Abläufe im ärztlichen Alltag verändern.

Die Digitalisierung wird daher auch das Arzthaftungsrecht nicht unberührt lassen und neue Fragestellungen aufwerfen, welche die Jurisprudenz der kommenden Jahre entscheiden und weiterentwickeln wird. Wahrscheinlich werden zukünftig die Sorgfaltsverpflichtungen der Ärzte eine neue Gestalt erhalten, auch im Hinblick auf nun mögliche Fernbehandlungen. Denkbar ist zudem, dass Patienten unter haftungsrechtlichen Gesichtspunkten selbst eine höhere Mitverantwortlichkeit erfahren werden.

Digitalisierung schenkt somit auch der Gesundheitswirtschaft die Chance, Kommunikation aktiv und effizient zu betreiben. Gesetze, Regeln und frühere Beschränkungen sind aufgehoben. Vernetzte Daten erschließen neue Dimensionen der Kommunikation und Beziehungen zwischen Behandler und Patienten.

\section{Interessenkonflikt}

Der Autor erklärt, dass keine wirtschaftlichen oder persönlichen Verbindungen bestehen.

\section{Autor}

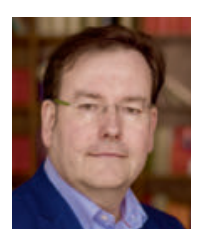

\section{Prof. Hans-Hermann Dirksen}

ist Hochschullehrer und Rechtsanwalt bei LIEBENSTEIN LAW - Kanzlei für Wirtschaftsrecht in Frankfurt am Main. Prof. Dirksen hat sich auf das Recht der Digitalisierung spezialisiert und berät bei der Vertragsgestaltung und Gesellschaftsgründung im Bereich Intellectual Property und Neue Technologien. In diesem Bereich ist er auch publizistisch tätig sowie ein gefragter Redner auf Konferenzen. Seit 2015 ist er Professor für Digitalisierung und lehrt an der FOM - Hochschule für Oekonomie und Management.

\section{Prof. Dr. Hans-Hermann Dirksen}

LIEBENSTEIN LAW - Kanzlei für Wirtschaftsrecht

Eschersheimer Landstr. 351

60320 Frankfurt am Main

E-Mail: mail@liebenstein-law.de

Internet: www.liebenstein-law.de

\section{Literatur}

[1] änd. änd-Umfrage zur Digitalisierung. 2018; Im Internet: https://www.aend.de/article/187896 (Abruf 26.08.2019)

[2] DAK-Gesundheit. DAK-Digitalisierungsreport 2018: So denken Ärzte über E-Health-Lösungen. 2018; Im Internet: https://www.dak.de/dak/download/dak-digitalisierungsreport-2018-1959528.pdf (Abruf 26.08.2019)

[3] Deutsche Apotheker- und Ärztebank. Digitalisierung im Gesundheitsmarkt - was Patienten sich wünschen, Ergebnisse einer Onlinebefragung von apoBank und Statista. 2018; Im Internet: http://newsroom.apobank.de/pressreleases/ umfrage-wieviel-digitalisierung-wollen-patienten-2485501 (Abruf 26.08.2019)

[4] Veit K, Wessels M, Deiters W. Gesundheitsdaten und Digitalisierung - Neue Anforderungen an den Umgang mit Daten im Gesundheitswesen. In: Pfannstiel MA, Krammer S, Swoboda W, Hrsg. Digitale Transformation von Dienstleistungen im Gesundheitswesen VI. Wiesbaden: Springer; 2019: 19-34

[5] Jameda. Studie: Arzt\&Digitalisierung. 2017; Im Internet: http://e-health-com.de/fileadmin/user_upload/dateien/Downloads/Studie_Arzt-und-Digitalisierung.pdf (Abruf 26.08.2019)

[6] Kassenärztliche Bundesvereinigung. Praxisbarometer Digitalisierung. Stand und Perspektiven der Digitalisierung in der vertragsärztlichen und psychotherapeutischen Versorgung. 2018. Im Internet: https://www.kbv.de

[7] kma Online. Arzthaftung bei telemedizinischem Angebot (Quelle: Prof. Jan Eichelberger). 2019; Im Internet: https://www.kma-online.de/aktuelles/medizin/detail/arzthaftung-bei-telemedizinischen-angeboten-a-40358 (Abruf 26.08.2019)

[8] Marstedt G. Das Internet: Auch Ihr Ratgeber für Gesundheitsfragen? Bevölkerungsumfrage zur Suche von Gesundheitsinformationen im Internet und zur Reaktion der Ärzte. 2018; Im Internet: http://www.bertelsmann-stif-tung.de/ fileadmin/files/BSt/Publikationen/GrauePublikatio-nen/ VV_Studie_Das-Internet-auch-Ihr-Ratgeber_Befragung.pdf (Abruf 26.08.2019)

[9] Wienke A, Fries K. Rechtliche Aspekte der Digitalisierung in der Medizin. Laryngo-Rhino-Otologie 2018; 97: 713-716

[10] Kulzer B. Arzt-Patienten-Beziehung: Im digitalen Zeitalter grundlegend verändert. Dtsch Arztebl 2015; 112(43): [20]; DOI: 10.3238/PersDia.2015.10.23.06

[11] OLG Oldenburg vom 27.05.2009 - 5U 43/08

[12] OLG Brandenburg vom 27.03.2008 - 12 U 239/06

[13] BGH 15.06.2010 - VI ZR 2047/09

[14] §7 Abs. 4 Berufsordnung der Ärzte Hessen

Bibliografie

DOI https://doi.org/10.1055/a-1016-6410

EHK 2019; 68: 265-269

(c) MVS Medizinverlage Stuttgart GmbH \& Co. KG ISSN 0014-0082 